\title{
Dynamique des ressources naturelles dans le Parc national de Manda: Cartographie et analyse pour le Développement durable
}

\author{
Solkam Rosalie Ballah, NDOUTORLENGAR Médard
}

\begin{abstract}
Abstrat: Au Tchad, le réseau d'aires protégées couvre près de 10,2\% de la surface du pays et reste globalement représentatif de toute la diversité des écosystèmes de la région. Cependant, ce réseau n'est pas constitué d'écosystèmes intacts car de nombreuses altérations y ont été apportés (certaines espèces sont déjà au seuil critique d'extinction (Addax, gazelle dama, lamantin), voire ont disparu (Rhinocéros noir et blanc, Oryx)) surtout dans les parcs nationaux. Ce qui nous amène à nous interroger sur la dynamique des ressources naturelles et le degré de conservation du parc national de Manda? Une évaluation de la diversité biologique et des ressources hydrographiques de 1951 à 1999 sur la base de la bibliographie existante, de la carte topographique de 1956, des images satellitaires Landsat 5 et 7 TM et ETM+ de 2 périodes (1986, 1999), complétée par des interviews semi-structurés et des transects sur le terrain, permettra de mieux appréhender la dynamique des ressources et les actions de conservation de la biodiversité réalisées à cet effet. Les résultats montrent une dynamique progressive de la faune de 1951 à 1970, puis une dynamique régressive de 1970 à 1989. Après cette tumultueuse période, un repeuplement du parc s'opère de 1989 à 2002. Par contre, la flore est relativement bien conservée avec quelques cours d'eau, des mares, champs et plantations. Et cela grâce aux multiples projets de conservation de la biodiversité. La promotion de l'écotourisme serait une alternative au développement durable de ce parc.
\end{abstract}

Keywords: Biodiversité, Gestion des ressources naturelles, Aire protégée, Parc national.

\section{Introduction}

Les ressources naturelles, plus particulièrement les aires protégées, sont devenues une préoccupation majeure de la fin du 20eme siècle tant par les mobilisations qu'elles suscitent que par les enjeux qu'elles sous-tendent. D'après la liste rouge publiée par l'Union Internationale pour la Conservation de la Nature (UICN) en 2015, sur les 79837 espèces étudiées, 23250 sont menacées, soit, $41 \%$ des amphibiens, $13 \%$ des oiseaux et $25 \%$ des mammifères, menacés d'extinction au niveau mondial ainsi que $31 \%$ des requins et raies, 33\% des coraux constructeurs de récifs et $34 \%$ des conifères. Le Programme des Nations Unies pour l'Alimentation et l'Agriculture (FAO) citée par Bergonzini et Lanly (2000) quant à elle, projette que le quart de la biodiversité de la planète risque de disparaître d'ici 2020 si rien n'est fait. Mais, comment préserver cet environnement tout en assurant les besoins essentiels de l'homme? Un moyen très efficace, mais aussi très radical de conserver la biodiversité d'un territoire terrestre ou aquatique selon les écologiques est de le doter d'un réseau d'aires protégées soustraites aux interventions humaines les plus perturbatrices. La plupart des pays Africains ont abordé cette question en intensifiant leurs efforts dans ce sens.

$\mathrm{Au}$ Tchad, le réseau d'aires protégées couvre près de $10,2 \%$ de la surface du pays et reste globalement représentatif de toute la diversité des écosystèmes de la région. On distingue du Nord au Sud, 2 parcs nationaux, 7 réserves de faunes, 2 domaines de chasse, 1 réserve de la biosphère, plusieurs forêts classées et réserves communautaires (Cf. figure $\left.\mathrm{n}^{\circ} 1\right)(\mathrm{H}, 1997)$. En revanche, ce réseau n'est pas constitué d'écosystèmes intacts car de nombreuses altérations y ont été apportés (certaines espèces sont déjà au seuil critique d'extinction (Addax, gazelle dama, lamantin), voire ont disparu (Rhinocéros noir et blanc, Oryx)). La biodiversité, demeure encore relativement riche, et recèle de nombreuses espèces présentes dans les parcs nationaux. Cependant, le niveau de classement et de suivi des différentes aires protégées n'est pas suffisant pour assurer la protection à long terme de ces écosystèmes (UICN/PACO, 2008). C'est le cas du parc national de Manda qui souffre de la pression pastorale, du braconnage, de la pêche illicite, de la pression démographique etc.

Ce travail est une Analyse diachronique des ressources du parc à partir de la carte topographique de 1956, des images satellitaires Landsat 5 et 7 TM et ETM+ de 2 périodes (1986, 1999), des transects et de la bibliographie existante. Les résultats montrent une régression de la faune, tandis que la flore est relativement bien conservée.

\section{Outils et méthodes}

Pour réaliser ce travail, nous avons adopté une méthodologie à deux niveaux : la première est la collecte des données basée sur la recherche bibliographique et les enquêtes de terrain dont les résultats sont traités et analysés selon la technique de l'analyse spatiale des images spatiales.

Après des recherches bibliographiques à Yaoundé et au Tchad dans les bibliothèques et institutions spécialisées pour recueillir la documentation sur la thématique, nous 
avons réalisé les entretiens sémi-structurés et des transects sur le terrain du 4 au 22 novembre 2007. Ces entretiens sont des enquêtes sommaires réalisées à l'aide d'un questionnaire adressé aux populations de Manda, Djoli, Miarom et Waïn sur l'exercice de leur activités socio-économiques, aux chefs de villages sur le mode de gestion du milieu, aux agents de conservation sur le mode de surveillance du parc, enfin aux ONG sur leur mode d'intervention dans la zone. L'échantillon est constitué de 52 individus correspondant à $100 \%$ de la population des 4 villages enquêtés.

Quant aux transects, nous avons relevé les coordonnées géographiques des activités humaines telles que les champs, les campements des pécheurs, des mares temporaires comme permanentes à l'aide d'un GPS de type Garmin 12 XL. Puis, nous avons réalisé 5 transects (à Nguére, Nangda, Djoli, Talia, et Waïn). Le positionnement s'est fait de deux manières. La première est la détermination de l'entrée du point au GPS en moto ou véhicule. Une fois le repère déterminé, on se dirige à l'intérieur à pied pour atteindre le point du transect. La seconde manière a consisté en la délimitation de la surface de $100 \mathrm{~m} 2 \mathrm{x} 100 \mathrm{~m} 2$ et la mesure des coordonnées des angles afin de définir des unités paysagères pour l'identification, la comparaison et la confirmation du type de végétation. La détermination de la flore s'est fait par une marche séquentielle pédestre avec l'aide d'un herbier local (Sara) et grâce à un guide chargé de chasse, M. Mahamat Koidegue accompagné de ses agents.

\section{II.2.Traitement et analyse des données}

L'analyse et le traitement des informations sur les images satellitaires et la carte topographique sont confrontées aux données de terrain pour mieux apprécier la dynamique des ressources naturelles. Pour le traitement des émulsions photographiques, nous avions tenu compte d'un certain nombre de paramètres propres aux techniques de prise de vue Tels que : le format de la photo, l'émulsion photographique utilisée, la période de prise de vue et l'échelle de prise de vue. Aussi, l'analyse de la réflectance des objets permet d'observer qu'elle varie pour un objet donné selon la longueur d'onde lumineuse ou la couleur dans le visible RATNAN N. 2005). L'émulsion Infrarouge Couleur (IRC) est celle utilisée dans nos images pour distinguer la composition et l'état de la végétation, l'hydrographie, etc.

Pour notre étude, nous nous sommes intéressés aux phénomènes généraux avec comme caractéristique dominante l'abondance de la végétation. Cependant, il faut tenir compte de la surface occupée par l'aire protégée pendant les différentes périodes pour relativiser les phénomènes. Ainsi, pour les transects, les travaux de cartographie d'occupation des sols ont été réalisés à une échelle : de 1/200000 (Niellim NC-33-XII et Sarh NC34-VII) pour l'ensemble de la zone couverte par deux images satellitaires Landsat 5 : TM et ETM couleurs (80 $000 \mathrm{~km}^{2}$ ). Sous le logiciel ENVI 4.0, des compositions colorées de plusieurs canaux Rouge (bande 4) Vert (bande 3) et Bleu (bande 2), avec le Moyen Infrarouge
(MIR) et le Proche Infrarouge (PIR) très sensible à la végétation, ont été réalisées. Ainsi, cinq (5) sur (11) transects prévus ont été réalisés selon la méthode d'échantillonnage systématique (premières unités choisies de manière aléatoire) pour effectuer des relevés phytoécologiques.

En l'absence du logiciel de traitements statistiques des données, nous avons effectué manuellement le calcul de la fréquence absolue des espèces végétales. Suivant le principe de l'abondance-dominance 70 espèces ont été déterminé dans les 5 transects. Enfin, la cartographie de ces espèces est faite sous le logiciel Mapinfo 7.8. Les espèces animales sont regroupées en troupeaux, leur nombre est systématiquement déterminé et classé suivant les différentes périodes (années). Ce qui a permis de déterminer les écarts absolus, le taux d'évolution. Il faut remarquer qu'aucun animal n'a été repéré dans le transect. Cependant, des Écureuils ont été observés tout le long du passage Nguere-Niellim dans le parc ainsi que des habitats d'oryctérope.

\section{LES RÉSULTATS ET DISCUSSIONS}

\subsection{Caractéristique du Parc national de Manda}

Le Parc National de Manda est compris entre le $9^{\circ} 20^{\prime}$ et le $9^{\circ} 50^{\prime}$ de latitude Nord et le $17^{\circ} 45^{\prime}$ et le $18^{\circ} 20^{\prime}$ de longitude Est. Il est limité au Nord par les souspréfectures de Baltoubaye et Korbol, au Sud par les souspréfectures de Moussafoyo, Béssada, Bédaya et Goundi, à l'Est par celles de Kotongoro et Bohobé, enfin à l'Ouest par celles de Goundi et Ndam (Ouyo B., 2005). Il est désenclavé par la route National N'Djaména-Sarh dans sa partie méridionale et le fleuve Chari dans sa partie Septentrionale.

L'article 2 du décret n56/EFPC du 19 mars 1956 (EFPC, 1956) stipule que le Parc National de Manda est créé pour "la propagation, la protection et la conservation de la vie animale sauvage et végétale sauvage dans un intérêt scientifique et éducatif au profit et à l'avantage et pour la recréation du public".

D'une superficie de 104.000 ha, la réserve a été consacrée initialement à la protection de certaines espèces dont l'élan de derby (Cf. figure 2 ). Le décret $\mathrm{n}^{\circ} 56$ a été suivi en 1966 après les indépendances d'un déguerpissement quasi total des villages qui se trouvaient à l'intérieur de l'aire protégée (les villages Koutou et Djoli sont encore restés en partie), sans indemnisation proprement dite. Le 23 octobre 1967, un nouveau décret $n^{\circ} 243 / \mathrm{PR} / \mathrm{PNR}$ vient étendre le parc à 114.000 ha allant jusqu'aux Rochers de Niellim pour protéger en plus de l'élan de Derby, le grand Koudou et d'autres espèces (Ballah solkam R, 2006).

La zone périphérique entourant immédiatement l'aire protégée regroupe 51610 habitants répartis dans quatre cantons (Abdelmadit et al, 1999) sans compter les éleveurs transhumants et les pécheurs saisonniers. Les principales activités menées par cette population sont: l'agriculture, la pêche, l'élevage bovin et de petits ruminants ainsi que la chasse "clandestine". L'organisation de l'espace autour du parc relève de 
l'autorité du chef de terre qui distribue les terres, organise les rites traditionnels et arbitre les litiges entre les exploitants. L'autorité administrative ne s'y mêle qu'en cas de litige grave ou conflit grave. Les zones non défrichées sont par excellence le domaine de la chasse ou de la cueillette collective.

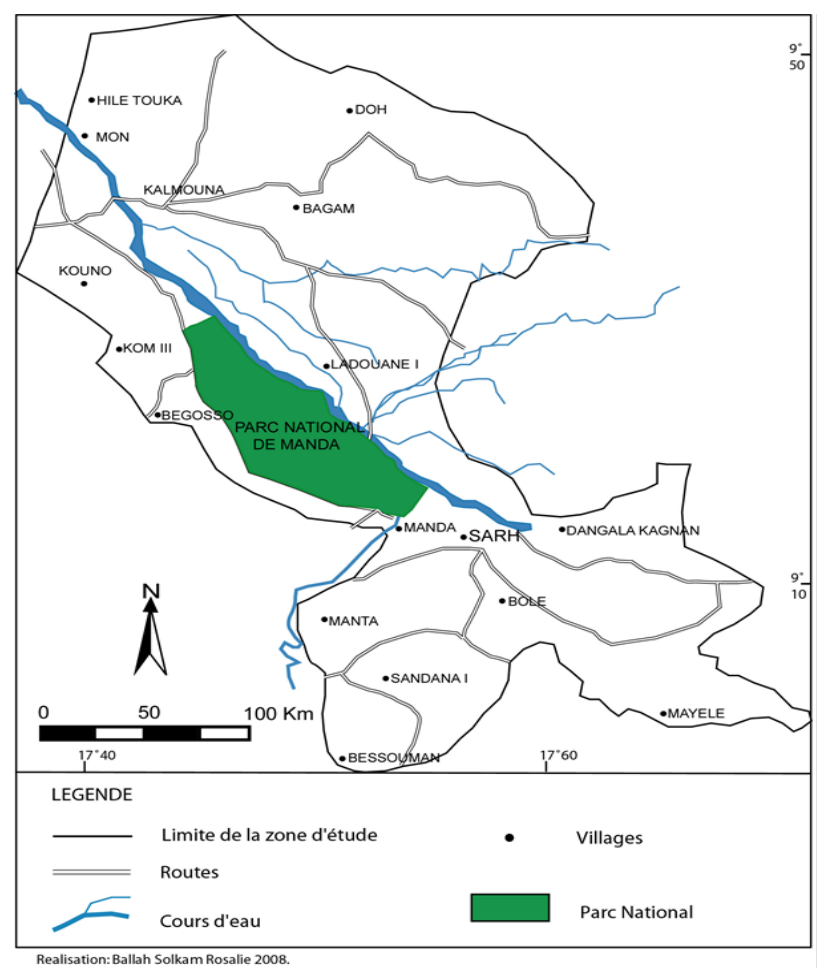

Figure 2: Localisation de la Zone d'étude

Source: Atlas PRASAC 2003

Ainsi, les "forêts", le fleuve Chari, les rivières, etc. constituent le domaine d'usage collectif à l'exception de ceux qui sont des œuvres humaines (plantés ou creusés), gérées par les propriétaires. Le statut actuel du parc interdit toutes activités dans l'enceinte de l'aire protégées à l'exception des rites traditionnels supervisés par les responsables du parc (FORETS, 1963).

En dehors de cette aire, les ressources telles que, les fleuves et forêts sont exploités sur permis (permis de chasse et permis de pêche), plus des redevances coutumières aux autorités traditionnelles (droit coutumier en guise d'impôt). Ce qui est un coup dur pour la population, et entraine souvent des conflits entre population et agents de l'État de protection du parc.

Soucieux de préserver l'environnement tout en assurant la survie des populations, le Gouvernement tchadien a ratifié en 1994 la Convention Internationale sur la Biodiversité. Cette convention a permis de mettre en place une politique de conservation qui vise : entre autres la conservation de la biodiversité, l'utilisation durable de ses éléments et le partage juste et équitable des avantages découlant de l'exploitation des ressources génétiques.

Dès lors, divers projets ont vu le jour. Nous pouvons citer entre autre, le Projet de Conservation de la Biodiversité durable dans le Moyen Chari, le projet «Interaction Élevage/Faune Sauvage» les actions de l'association pour la protection et le développement de la pisciculture intégrée au Tchad (APRODEPIT) et de l'Association pour la Promotion des Initiatives Communautaires en Afrique (APICA), Pour ne citer que ceux-là. Ces projets prônent la gestion durable des ressources naturelles et cogestion entre les populations et tous les autres acteurs impliqués dans la gestion du parc.

\subsection{Dynamique des ressources autour du PNM}

\subsubsection{Un élevage transhumant qui se sédentarise autour du parc}

L'ONDR fournit pour le Moyen Chari en 1998 des chiffres de bétail dont 200.000 de bovins, 125.000 d'ovins et 300.000 de caprins, mais ce recensement n'inclut que les animaux des sédentaires.

\begin{tabular}{|c|c|c|c|c|c|c|c|c|}
\hline & 1997 & & & & 1998 & & & \\
\hline $\begin{array}{l}\text { Cantc } \\
\text { ns }\end{array}$ & $\begin{array}{l}\text { Bovi } \\
\text { ns }\end{array}$ & Ovins & $\begin{array}{l}\text { Capri } \\
\text { ns }\end{array}$ & $\begin{array}{l}\text { Vola } \\
\text { lle }\end{array}$ & $\begin{array}{l}\text { Bovi } \\
\text { ns }\end{array}$ & Ovins & $\begin{array}{l}\text { Capri } \\
\text { ns }\end{array}$ & $\begin{array}{l}\text { Volail } \\
\text { le }\end{array}$ \\
\hline $\begin{array}{l}\text { Balin } \\
\text { ba }\end{array}$ & $\begin{array}{l}1529 \\
3\end{array}$ & 12493 & 21540 & 0 & $\begin{array}{l}1865 \\
5\end{array}$ & 1478 & 33292 & 29130 \\
\hline $\begin{array}{l}\text { Koka } \\
\text { ga }\end{array}$ & 0 & 0 & 0 & 0 & 0 & 0 & 0 & 0 \\
\hline Djoli & 2262 & 0 & 4954 & 0 & 4879 & 1865 & 6314 & 11480 \\
\hline $\begin{array}{l}\text { Nielli } \\
\text { m }\end{array}$ & 4954 & 646 & 6785 & 0 & 5309 & 2009 & 7462 & 11623 \\
\hline Total & $\begin{array}{l}2250 \\
9\end{array}$ & 13139 & 33279 & 0 & $\begin{array}{l}2884 \\
3\end{array}$ & 5352 & 47068 & 52233 \\
\hline
\end{tabular}

Tableau 1: Évolution du cheptel par canton autour du parc.

Source: Office National de Développement Rural, 1998

NB : Les données du canton Kokaga n'ont pas été fournies.

On constate d'après le tableau une augmentation continue de l'élevage autour du parc. En dehors du bétail transhumant, le nombre des bovins est passé de 22509 têtes en 1997 à 28843 têtes en 1998, tandis que l'élevage des ovins diminue. L'élevage des Caprins, lui, a augmenté à 13789 de têtes en 1998. Le taux annuel du cheptel de ruminants est estimé par la Direction de L'Élevage et des Ressources Animales (DERA) à 2,4\% par an. En septembre 1998, une étude faite par le CIRAD-EMVT estimait la vente un bœuf adulte à $58.000 \mathrm{Fcfa}$, un taureau adulte à $63.000 \mathrm{Fcfa}$, démontrant ainsi l'importance de ce secteur pour la population (Ballah Solkam R, op.cit).

\subsubsection{La pêche, une activité de plus en plus sollicitée}

Le Moyen Chari est une zone très riche en ressources halieutiques en raison du nombre de cours d'eau qui le traversent (le Chari, le Bahr Kôh, le Bahr Sara, le Bahr Salamat etc.). Les pêcheurs viennent de partout. Ils font des navettes entre ces différentes cours d'eau en période d'activité de juin à octobre et repartent vers novembre à leurs résidences en période d'étiage. Ils utilisent pour la plupart des sennes de plage et des éperviers prohibés par les lois pour pêcher. Cependant, des campagnes de sensibilisation et de formation opérées par APRODEPIT en technique de production ont été très positives dans le secteur de pêche (Sanni N. 1999). On trouve un peu partout des pêcheurs en charge d'exploitation des étangs 
de pisciculture extensive tels que le groupement Lapamou à Waïn qui aménage des bras du fleuve Chari et des mares. A la décrue, la pêche illégale s'accentue dans le parc (Ballah Solkam R, 2008).

Les espèces de poissons prélevées varient du Lattes Niloticus (capitaine) au Alestes dentex (sardinelles photo 3 et 4). Le poisson est vendu frais, fumé ou séché. En une saison, un pêcheur peut collecter 100 sacs de petits poissons. Un sac coûte entre 12.000 - 35000 Fcfa selon les périodes, plus $600 \mathrm{Fcfa}$ de taxes du service des eaux et forêts et des $1000 \mathrm{Fcfa}$ de frais de transport du lieu de pêche jusqu'à Moundou (Ramadji N. op.cit). En dehors de ces taxes, le pêcheur verse une certaine redevance de 50.000 Fcfa avant toute activité de pêche au Mbang de Bédaya et $25.000 \mathrm{Fcfa} / \mathrm{mois}$ au chef canton de Kokaga (Tchago B.1999).
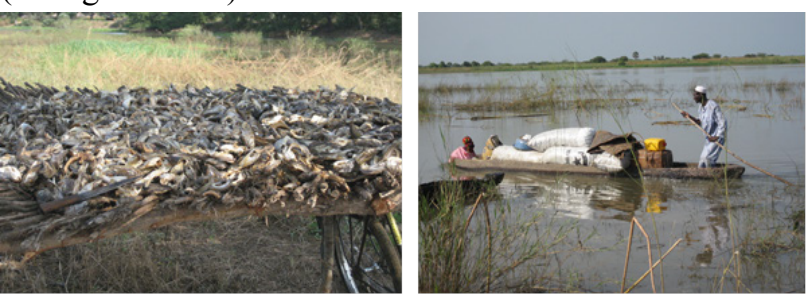

Figure 3: Sardinelles séchées et transportées vers Sarh

Source : Ballah Solkam Rosalie, novembre 2007

Les 2 photos montrent les techniques de séchage et le transport de poissons vers Sarh pour la commercialisation. De là, les commerçants viennent en acheter pour aller les revendre à Sarh, Moundou et autres villes. Selon les estimations, le secteur de pêche du Moyen Chari compte 110 pêcheurs professionnels qui exercent à temps plein (estimation faite sur le nombre des permis de pêche délivrés). La production de tout le moyen Chari dépasserait 11.500 tonnes pour les 9 premiers mois de l'année 1998 (CIRAD-EMVT, 1999).

\subsubsection{La chasse, une activité qui devient dangereuse}

La chasse autorisée se pratique en dehors des parcs nationaux, dans les zones d'intérêts cynégétiques du Moyen Chari. Mais depuis les événements malheureux que la région a connu, ces zones sont peu fréquentées par les touristes étrangers. Par conséquent, c'est la chasse traditionnelle qui est développée avec le braconnage non sélectif qui constitue un danger pour les espèces animales indispensables à la régulation écologique. Les espèces les plus braconnées sont : le rhinocéros, la panthère, l'oryctérope, le porc-épic, les canards et les pintades comme le montre la figure 4 entraînant une perte des recettes dans l'économie nationale. Mais, les activités de réhabilitation du parc initiées par le Fond d'aide et de Coopération (FAC) connaissent un début prometteur pour réveiller ce secteur (Ballah Solkam R, op.cit.).
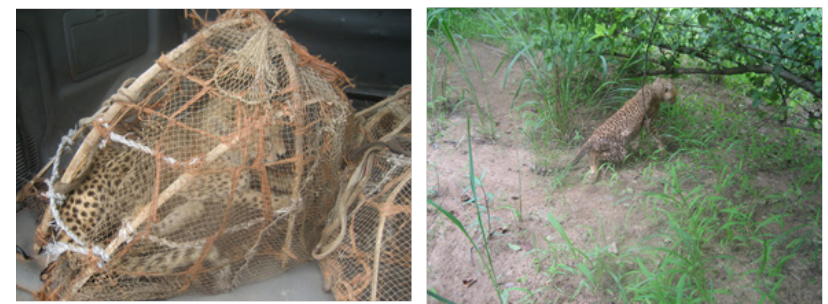

Figure 4: Des panthères braconnées, récupérées et réintroduites dans le parc

Source : Projet Biodiversité 2007

\subsubsection{Les coupes de bois et leurs impacts sur l'environnement}

Les ménages autour du parc ont recours aux bois de chauffe prélevés à l'intérieur ou à l'extérieur dudit parc pour la satisfaction de leurs besoins énergétiques et des besoins de construction. C'est le cas du Khaya senegalensis, d'Isoberlinia doka et d'Anogeissus leiocarpus très sollicitées pour leur résistance à l'eau. La proximité de la ville de Sarh exerce une grande influence sur le fonctionnement de cette activité, car $98 \%$ des ménages du Moyen Chari, utilisent les combustibles ligneux (Sanni N. op.cit). Pour des raisons économiques, même les espèces fruitières telles que : Vitelleria paradoxa et Tamarindus indica sont aujourd'hui sacrifiées. Un autre fait inquiétant est que pour nourrir leurs bêtes, les éleveurs coupent ou déracinent carrément certaines espèces (Ballah Solkam R, op.cit.).
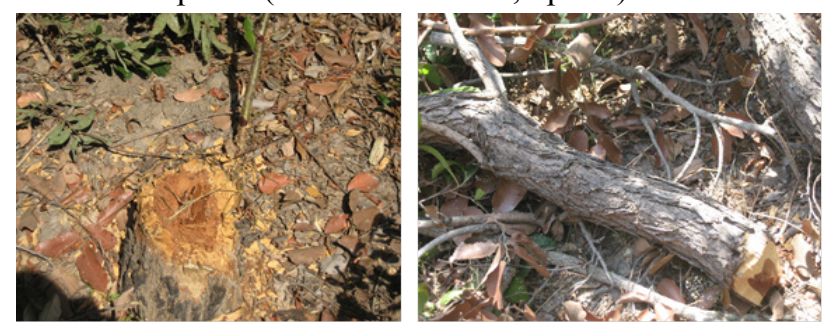

Figure 5: L'Isoberlinia doka coupé dans le parc

Source : Ballah Solkam Rosalie, novembre 2007

\subsection{Cartographie des ressources naturelles dans le parc}

La dynamique autour du PNM n'est pas sans conséquence sur le parc lui-même. L'évolution de la faune du parc est lamentable du point de vue quantitatif, tandis que la flore est relativement bien conservée. Les ressources hydrographiques quant à elles ont connu aussi une régression considérable. Selon les estimations de M. et Mme Sommer (François, 1970), la dynamique de la faune du parc depuis sa création en 1951 à 1970 est progressive. Cependant les observations selon d'Abdoulaye en 1981 et de PFEFFER (Pierre, 1993) confirmées récemment par celles de Lamarque et DEJACE montrent que cette dynamique régressive.

\begin{tabular}{|l|c|c|c|c|c|c|} 
& $\mathbf{1 9 5 1}$ & $\mathbf{1 9 6 5}$ & $\mathbf{1 9 7 0}$ & $\begin{array}{c}\mathbf{1 9 8} \\
\mathbf{1}\end{array}$ & $\mathbf{1 9 8 9}$ & $\begin{array}{c}\mathbf{1 9 9} \\
\mathbf{6}\end{array}$ \\
\hline Autruches & $\begin{array}{c}\text { quelqu } \\
\text { es }\end{array}$ & $\begin{array}{c}\text { quelqu } \\
\text { es }\end{array}$ & $\begin{array}{c}\text { quelqu } \\
\text { es }\end{array}$ & 10 & 0 & 35 \\
\hline
\end{tabular}




\begin{tabular}{|c|c|c|c|c|c|c|}
\hline Bubales & 55 & 250 & 900 & - & 0 & 18 \\
\hline \begin{tabular}{|l|} 
Buffles \\
\end{tabular} & 250 & 350 & 800 & - & $\begin{array}{l}\text { une } \\
\text { trace }\end{array}$ & $\begin{array}{l}\text { des } \\
\text { trac }\end{array}$ \\
\hline $\begin{array}{|ll|}\text { Cobes } & \text { de } \\
\text { Buffon } & \\
\end{array}$ & 150 & 950 & 3250 & - & 25 & 90 \\
\hline $\begin{array}{l}\text { Cobe } \\
\text { défassa }\end{array}$ & 225 & 650 & 5000 & 125 & $\begin{array}{c}\text { quelqu } \\
\text { es }\end{array}$ & 38 \\
\hline \begin{tabular}{|l|} 
Crocodiles \\
\end{tabular} & - & - & - & - & - & 5 \\
\hline $\begin{array}{l}\text { Cynocéphal } \\
\text { es }\end{array}$ & - & - & - & - & - & 90 \\
\hline Damalisque & 18 & 43 & 125 & 0 & 0 & 8 \\
\hline \begin{tabular}{|ll}
$\begin{array}{l}\text { Élan } \\
\text { derby }\end{array}$ & de \\
\end{tabular} & 135 & 450 & 900 & 40 & 0 & $\begin{array}{l}\text { une } \\
\text { trac }\end{array}$ \\
\hline 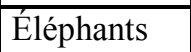 & 65 & 350 & 1750 & 50 & 0 & 0 \\
\hline Girafes & - & $\begin{array}{l}\text { quelqu } \\
\text { es }\end{array}$ & $\begin{array}{l}\text { quelqu } \\
\text { es }\end{array}$ & - & 0 & 0 \\
\hline $\begin{array}{l}\text { Guibs } \\
\text { harnachés }\end{array}$ & 35 & 125 & 200 & 75 & 45 & 80 \\
\hline $\begin{array}{l}\text { Hippopota } \\
\text { mes }\end{array}$ & $\begin{array}{c}\text { quelqu } \\
\text { es }\end{array}$ & 150 & 300 & 30 & $\begin{array}{l}\text { une } \\
\text { trace }\end{array}$ & 6 \\
\hline $\begin{array}{l}\text { Hippotragu } \\
\text { es }\end{array}$ & 100 & 135 & 500 & - & $\begin{array}{c}\text { quelqu } \\
\text { es }\end{array}$ & 35 \\
\hline $\begin{array}{l}\text { Hyènes } \\
\text { rayées }\end{array}$ & - & - & - & - & - & 13 \\
\hline Léopards & rares & 55 & 90 & 43 & 0 & 23 \\
\hline Lions & rares & 55 & 90 & 43 & 0 & 4 \\
\hline Lycaons & - & - & 65 & - & - & 18 \\
\hline $\begin{array}{l}\text { Phacochère } \\
\text { s }\end{array}$ & 75 & 700 & 2000 & 150 & 90 & 325 \\
\hline Réduncas & 20 & 175 & 200 & 125 & $\begin{array}{c}\text { des } \\
\text { traces }\end{array}$ & 50 \\
\hline
\end{tabular}

Tableau 2: Évolution estimée des effectifs moyennes de la grande faune de Manda

Source : synthèse des données de SOMMER, Abdoulaye, PEFFER, Lamarque

Les estimations de Sommer doivent être considérées avec beaucoup de prudence, car, certaines espèces telles que l'éléphant, l'élan de Derby, le buffle, etc. effectuent d'importants déplacements saisonniers et leur effectif dans une zone aussi limitée que le parc devaient varier suivant les périodes. Il est certain qu'entre les estimations de 1951 et de 1965 après 15 ans de mise en réserve, il y a eu une accoutumance des animaux avec la présence de l'homme. Le retour timide des animaux s'est opéré dans les années 1996 grâce au projet de réhabilitation et d'aménagement du parc financé par la coopération française en 1994 comme en témoins le graphique cidessous à l'exception des crocodiles, des cynocéphales, des hyènes rayée, dont on n'a pas pu déterminer, mais qui seront dénombrés en 1994 par la Coopération française (Ballah Solkam R, op. cit).

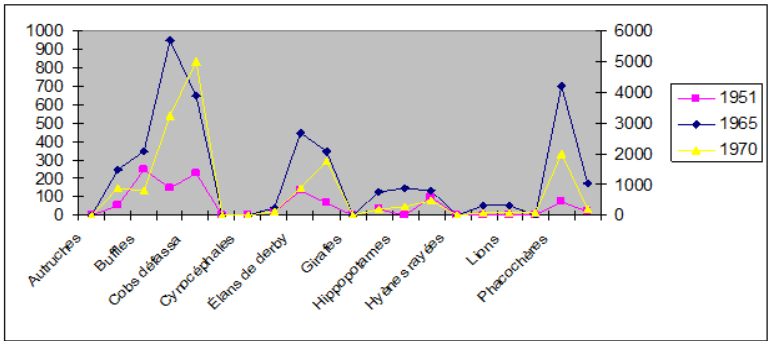

Figure 6 : Évolution progressive de la faune de 1951 à 1970

Source : synthèse des données de SOMMER, Abdoulaye, PEFFER, Lamarque

\subsubsection{Une régression de la faune de 1970 à 1989}

Le Parc de Manda hébergeait au début des années 1970 une faune abondante et variée. Les prélèvements "illicites" n'avaient pas provoqué au début des années 1970, un impact aussi visible qu'aujourd'hui (absence de la faune). Cette situation a évolué négativement compte tenu du processus de désertification et de la guerre civile de 1979. Les pâturages devenant rares autour du parc, les éleveurs transhumants s'y déversent pour faire paître leur bétail. Par ailleurs, le retour des rescapés de guerre a augmenté l'effectif de la population de la ville de Sarh qui puise l'essentiel de sa subsistance dans le parc (Ballah Solkam R, op. cit.).

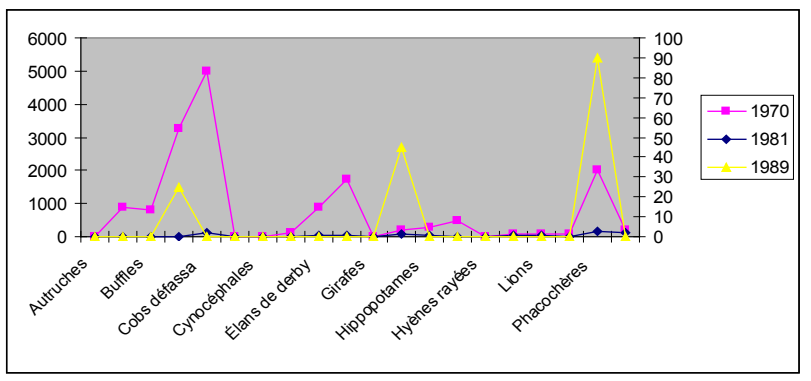

Figure 7: Evolution régressive de 1970 à 1989

Source : synthèse des données de SOMMER, Abdoulaye, PEFFER, Lamarque

\subsubsection{Puis, un repeuplement de 1989 à 2002}

Après une période de croissance et de décroissance relative, les animaux reviennent peu à peu dans le parc vers la fin des années 1989. C'est le cas de Cobes de Buffon, Phacochères, Guibs harnachés, Cynocéphales etc. en nombre croissant par rapport aux années 1980. En 1993, un pisteur du parc a déclaré à la mission PFEFFER qu'il aurait aperçu 5 élans de Derby en 1992. Les années 1990 apparaissent comme une année relativement calme pour les animaux. Ceci grâce aussi aux efforts de la Coopération Française. Ce calme a favorisé aussi l'émergence des espèces comme : le cobe de Buffon, le phacochère, le guib harnaché etc. qui ne sont pas déterminées dans les estimations du tableau. Les traces de certains animaux au cours des années 1995-96 sont des indices de vie très appréciables. Cependant, en 1998, la poursuite de la rébellion du Dr NAOR sur les collines de 
Niellim au nord du parc par l'armée nationale tchadienne aurait plongé de nouveau le parc dans le désastre en faisant fuir de nombreux animaux (Ballah Solkam R, op.cit).

Nous ne disposons pas de données récentes pour étayer notre argumentation sur l'état de la faune dans les années 2000. Cependant, les estimations d'APRODEPIT sur la base des fiches animalières mises à la disposition des agents de 1999 à 2000, permettent de faire des simulations. Par ordre croissant, les espèces suivantes ont été répertoriées en cette période : le phacochère, le cobe de Buffon, l'hypotrague, le guib harnaché, le cobe de roseau, l'hippopotame etc. On pourrait donc s'attendre à un peuplement massif de la zone et partant du parc avec la présence de nombreux éléphants signalés à Dembo si vraiment le projet biodiversité favorise la migration de la faune dans le corridor mentionné dans la plupart des documents. L'avifaune, quant à elle est très abondante et continue de se multiplier grâce au fleuve Chari.

3.3.3 Avec un maintien de la couverture végétale de la réserve au Parc National de Manda de 1951 à 1999

Les données sur l'occupation du sol en 1951 faisant défaut, nous nous sommes basés sur la carte topographique de 1956 et l'image satellitaire de 1986 et 1999 pour faire ce bilan. Si sur la carte topographique, nous avons fait recours á la légende, avec les images satellitaires, nous avons pris comme critère la teinte pour définir les différents phénomènes observés. En 1956, la réserve de Manda avait une superficie de 104.000 ha. La végétation était constituée de savane boisée qui occupait à peu près $70 \%$ de la surface du sol. Les espaces dégradées sont celles occupées par les champs et plantations (environ $8 \%$ ). On dénombrait 8 champs et 4 plantations. Les ressources hydrographiques quant à elles, occupent environ $30 \%$ de la surface, le Chari constitue le principal cours d'eau qui alimente les mares du parc. On compte environ 76 mares permanentes et 52 mares temporaires.

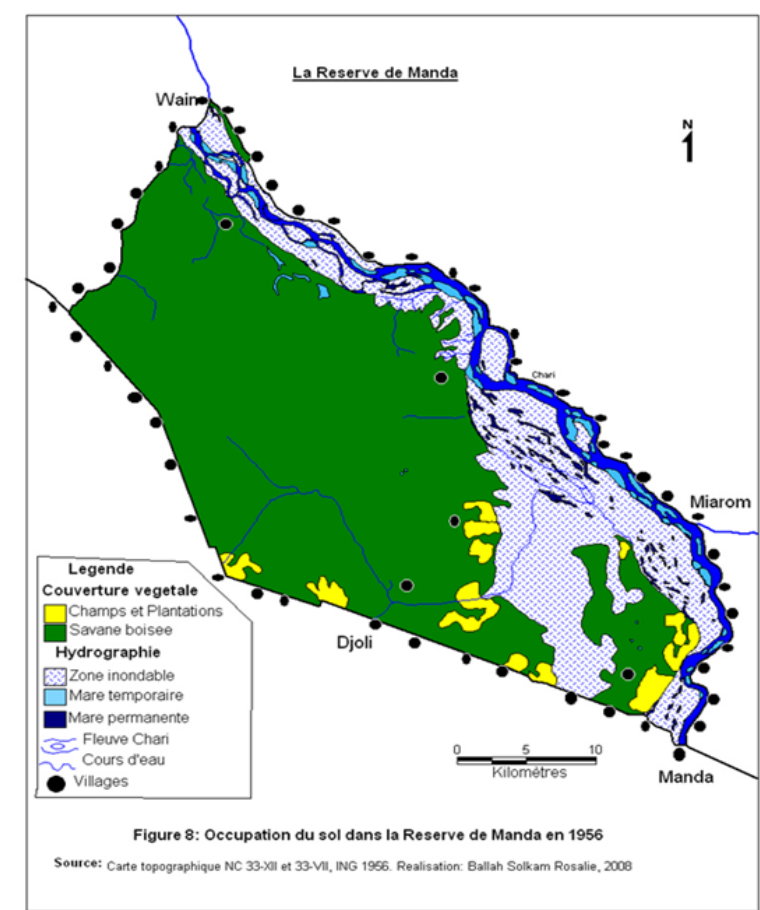

Des espaces importantes autour du Chari constituent ces zones d'inondations permanentes (environ 6\%). Trente ans après, c'est-à-dire en 1986, la superficie de la réserve devenue Parc de Manda passe à 114.000 ha (Ballah Solkam R, op.cit.).

L'image satellitaire Landsat TM du 9 décembre 1986 met en lumière les différents contrastes de végétation et les ressources hydriques. Elle montre que, la végétation reste toujours la savane boisée (environ 74\%) avec des zones de végétation active de forêts claires à Combretum collinum et detarium microcarpum (14\%), et des savanes arbustives à Oxytenatera abissinica et Detrarium microcarpum (7\%). Les ressources hydrographiques occuperaient $26 \%$ de la surface totale du parc. Le Chari reste toujours le principal cours d'eau alimentant le parc auxquels s'ajoute 43 mares permanentes et 20 mares temporaires même, si plusieurs cours d'eau traversent le parc. La zone inondable du Chari représenterait 33\%. Ce chiffre doit être pris avec beaucoup de précaution, car il est basé essentiellement sur les phénomènes visibles à l'échelle de l'image (Ballah Solkam R, op.cit.). 


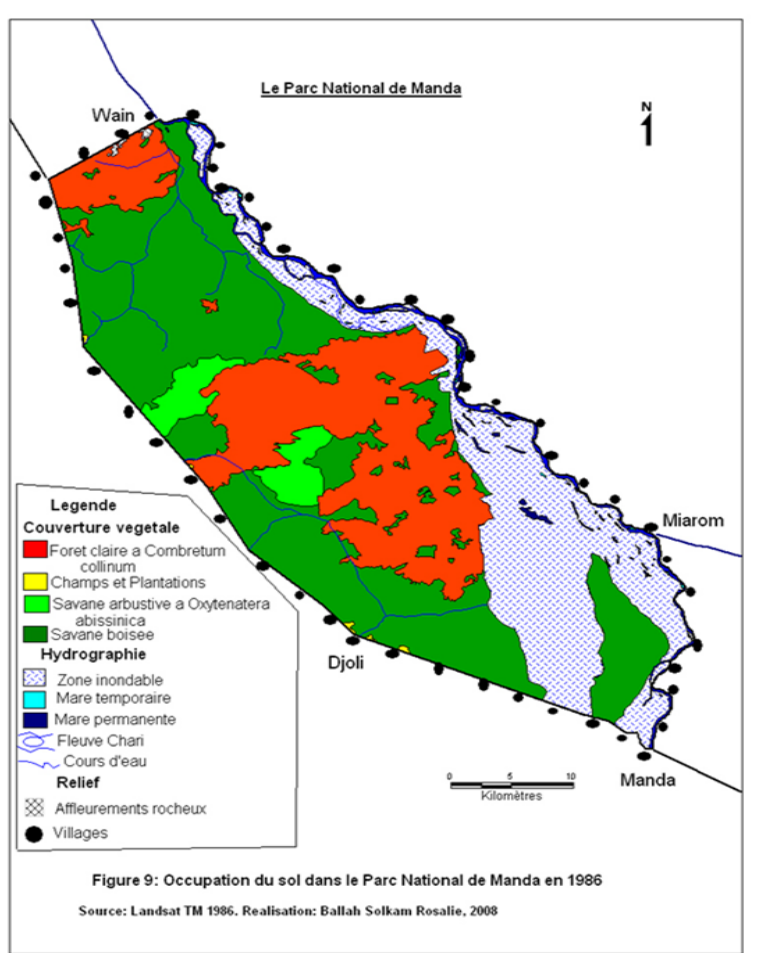

On observe aussi des collines au nord du parc à cause de l'extension de ce dernier pour préserver le Grand koudou. Par contre, la limite du parc en 1999 est la même qu'en 1986 mais, elle présente des spécificités (Ballah Solkam R, op, cit.).

Le format de l'image ETM+ du 3 novembre 1999 par contre, présente un contraste entre la végétation et l'eau. Ce qui montre l'étendue du Chari englobant toutes les zones inondables des alentours (figure 2). La végétation du parc en 1999 occuperait $68 \%$ de la surface totale du parc. Elle est composée de savane boisée et de savane arbustive toujours à Oxytenatera abissinica et Detarium microcarpum avec quelques champs et plantations au bord du parc.

L'image de 1999 ne montre pas une végétation très verdoyante comme celle de 1986. Les ressources hydrographiques sont à vue d'œil bien conservées aussi. Elles représenteraient $32 \%$ de la surface totale du parc. Ce pourcentage est important par rapport aux deux premières images parce qu'elle englobe les zones inondables du Chari.

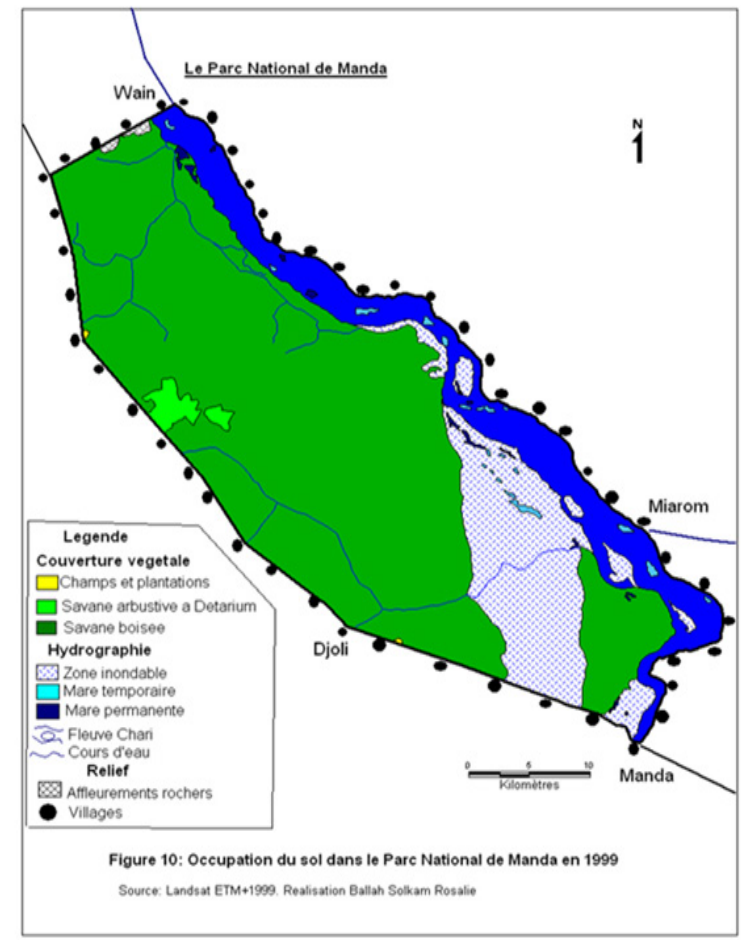

Cependant, les mares semblent toujours en régression, mais les cours d'eau traversant le parc demeurent permanents. Quelques 16 mares permanentes et 19 mares temporaires sont repérées. Comme nous l'avions dit cihaut, ces chiffres sont à prendre avec beaucoup de réserve pour ne pas biaiser les informations.

L'analyse de l'occupation du sol des deux périodes montre que malgré la pression démographique, la végétation est relativement bien conservée à l'exception de la faune qui est en régression, mais ce dernier pourrait repeupler le parc si les efforts convergent dans ce sens. La population peu nombreuse de départ a favorisé une croissance des espèces tant animales que végétales dans le parc entre 1951 à 1970 . Le braconnage s'en trouvait limité. La situation va se renverser après les années 1970 et 1980 à cause de la guerre civile et la sécheresse, elle va s'empirer dans les années 1990 avec la croissance démographique. Ce qui justifie la régression continue de la faune à ces périodes.

Après les années 1990, le calme semble revenir et les migrations se font de plus en plus vers la zone du Moyen Chari qui est soumise à de multiples et intenses pressions $\mathrm{du}$ fait de la croissance démographique traduit par une demande croissante de terres agricoles, de la sédentarisation des éleveurs ainsi que du nombre croissant de troupeaux en transhumance etc. Ces pressions font peser des menaces immédiates sur la diversité biologique tant dans les zones de terroirs que sur le Parc National de Manda. La série de sécheresse des années 1970 a contribué à une fragilisation de différentes formations végétales consécutives des écosystèmes sahélo-soudaniennes se traduisant, par endroits, par des mortalités sur pied des arbres, affaiblis par des passages répétés de feux de brousse ainsi que de la disparition de la biodiversité animale (Ballah Solkam R, op, cit.). 


\section{Conclusion}

Selon le projet biodiversité du Moyen Chari, la zone d'action du projet couvre l'une des dernières zones écologiques de savanes sahélo-soudaniennes comprenant un ensemble composé de biotopes variés. Ces biotopes hébergent une faune abondante et variée représentant à elles seules, environ $90 \%$ des 70 espèces mammifères et $70 \%$ des espèces d'oiseaux que compte le Tchad. La région est donc notamment réputée pour la présence d'une population d'espèces fauniques d'importance mondiale, vulnérables et menacées d'extinction, inscrites sur la liste rouge de l'UICN (1990). Ces espèces sont : le Damaliscus korrighum, Alcephalus buselaphus, Taurotragus derbianus, Gazella ruffifons, Gasella cuvieri, Gasella dama, Gasella dorcas, Gasella leptoceros, Oryx dammah, Ammotragus lervia, Loxodontus africana, Lycaon pictus. En dehors des espèces déjà recensées, le projet estime que la zone offre également des potentialités non négligeables pour divers mammifères et vertébrées dont il conviendrait de confirmer la présence. D'importantes populations d'oiseaux afro tropicaux et paléarctiques ont également pu être recensés dans le parc (Keith James O, 1997). Ces richesses représentent le patrimoine de l'Afrique subsaharienne qui mérite d'être protégées.

Il faut noter que les expériences du Parc de Manda ont démontré la nécessite d'instaurer une démarche concertée en vue de l'amélioration de sa gestion. La promotion de l'écotourisme serait une alternative au développement durable de ce parc.

\section{Bibliographie}

ABDELMADIT et al. (1999). Le parc national de Manda : écosystèmes pastoraux, Rapport d'étude, 36

BERGONZINI J-C et LANLY J-P, (2000). Les forêts tropicales, la Librairie du Cirad, Karthala, 164 P.

BALLAH, Solkam.R. (2006). La problématique de gestion du parc de Manda: Enjeux fonciers et Perspectives, Mémoire de maîtrise, Université de N'Djaména, 83 P.

BALLAH, Solkam.R. (2008). Le Parc national de Manda: dynamique des ressources naturelles et cogestion, mémoire de DEA, Université de Yaoundé1.

CIRAD-EMVT, (1999). Étude de faisabilité économique du projet integré de conservation communautaire de la biodiversité dans le Parc national de Manda et ses zones adjacentes, Montpellier France, $68 \mathrm{P}$.

DJERANG S., (1998). La conservation des aires protégées au Tchad. Rapport d'étude DESS. Limoges. $16 \mathrm{P}$.

DIRECTION DES EAUX ET FORETS, (1963). Ordonnance $n^{\circ} 14 / 63$ du 28 mars 1963 règlementant la chasse et la protection de la nature. $20 \mathrm{P}$.

EFPC. (1956). décret n56/EFPC du 19 mars 1956.

FAO. (2000). La foresterie participative : une stratégie pour une gestion durable des forets d'Afrique, pp 317 326. in Rapport de l'atelier de Banjul.
FAO. (2000). Les forêts tropicales dans le monde, la Librairie du CIRAD, Rapport 5 P.

FORETS, D. D. (1963). Ordonnance $n^{\circ} 14 / 63$ du 28 mars 1963 règlementant la chasse et la protection de la nature. $20 \mathrm{P}$.

KEITH James O. et PLOWES Darrel C. H, ( 1997). Considerations of Wildlife Resources and Land Use in Chad, Rapport de mission, 29 P.

Liste rouge . 67. http://www.delegfrance-onugeneve.org/blogindex.php?2006/05/105-liste

Maryland (2006) http://glcf.umiacs.umd.edu

OUYA Bondero, (2005). Conservation de la biodiversité et développement. Enjeux autour des aires protégées du sud-est du Tchad. Dynamique d'occupation et partage de l'espace : cas des parcs Nationaux de Manda et Zakouma, Mémoire de Master1, Université de Montpellier III, $110 \mathrm{P}$.

Office National de Développement Rural, 1998.

MENGUE-MEDOU Célestine, (2002). Les aires protégées en Afrique : perspectives pour leur conservation. Revue en sciences de l'environnement sur le WEB, Vol 3, $124 \mathrm{P}$

$\begin{array}{cccc}\text { MINISTERE DE L'ELEVAGE, } & \text { DE } & \text { LA } \\ \text { COMMERCIALISATION } & \text { DES } & \text { PRODUITS }\end{array}$ ANIMAUX, EAUX-FORETS, PECHE ET CHASSES. Ordonnance $\mathrm{n}^{\circ} 14 / 63$ du 28 mars 1963 Règlementant la Chasse et la Protection de la Nature. 37 P

MINISTÈRE DE L'ENVIRONNEMENT, (1999). Les aires protégées : Cadre d'orientation en vue d'une stratégie québécoise. $20 \mathrm{P}$.

PFEFFER, Pierre. (1993). Mission effectuée du 11 au 18 décembre 1993 pour le ministère de la coopération et du développement. Mission de coopération et d'action culturelle. Rapport, N'Djamena, 20

RAMADJI Nadjibaye N., (2004). Pression anthropique et problématique de la gestion du parc national de Manda: Bilan et Perspectives, Mémoire de Maîtrise, Université de N'Djaména, $98 \mathrm{P}$.

RATNANT Ngadoum, (2005) Analyse spatiale, Polycope, Université de N'Djamena, 67 P

SANNI G., (1999). Les rapports d'étude économique sur le parc national de Manda, N'Djamena, $45 \mathrm{P}$.

SOMMER François. (1970). Note technique sur le parc national de Manda. N'Djamena, République du Tchad. $35 \mathrm{p}$.

TCHAGO Bouimon., (1999). Les systèmes de gestion participative et rationnelle avec une exploitation et une gestion optimale des ressources par l'atténuation des pressions existantes dans et autour du parc national de Manda, Rapport d'étude, N'Djamena, 108 P. 